\title{
The Spatial Region of Photoionization in a Strong Laser Field
}

\author{
J.H. BAUER* \\ Katedra Fizyki Teoretycznej Uniwersytetu Łódzkiego \\ Pomorska 149/153, 90-236 Łódź, Poland
}

(Received July 2, 2008)

\begin{abstract}
In the framework of the Keldysh-Faisal-Reiss theory in the velocity gauge we investigate the magnitude of the space region, where photoionization in a strong laser field takes place. We find substantial differences between the short-range and the long-range (Coulomb) potentials, and between linear and circular polarizations of incident radiation. It appears that only for the initial state in the Coulomb potential the region of space, where ionization is held, expands significantly with increasing intensity for a typical optical frequency and non-relativistic but strong circularly polarized laser field. As a result of our considerations, we suggest to modify the idea of Reiss and Krainov of a certain simple Coulomb correction to the Volkov wave function. We show that photoionization rate calculated for the $\mathrm{H}(1 s)$ atom, using our approach, is in better agreement with other theoretical results for moderately strong circularly polarized laser field.
\end{abstract}

PACS numbers: $32.80 . \mathrm{Rm}, 42.50 . \mathrm{Hz}$

\section{Introduction}

In 1994 Reiss and Krainov published the paper entitled "Approximation for a Coulomb-Volkov solution in strong fields" [1], where the well-known Volkov (or Gordon-Volkov) wave function [2, 3], multiplied by a time-dependent exponential factor, has been used as a final state in the Keldysh-Faisal-Reiss (KFR) theory [4-8]. Although this Coulomb correction is remarkably simple (and resolves itself into a small shift of the binding energy of the $\mathrm{H}(1 s)$ atom), it can increase the calculated ionization rate by a few orders of magnitude (see, for example, Fig. 2 of [1]). One can also derive the Coulomb-Volkov wave function (Eq. (5) in the present paper) using the non-perturbative path-integral approximation $[9,10]$. One of the assumptions utilized in [1] is the following one: the most significant contribution to the transition matrix element should be given by the region of small electron-nucleus distances, because the atomic ground state is located there (see the text below Eq. (7) of [1]). It is the main purpose of the present paper to

*e-mail: bauer@uni.lodz.pl 
investigate validity of the statement more exactly. We will show that for ionization in a strong laser field the above mentioned region may be much greater.

Our paper is organized as follows. In Sect. 2 we derive strong-field ionization rates (in the version of Faisal-Reiss [6, 7]) in finite spatial region and compare them numerically with standard results. Based on the comparisons we propose new Coulomb correction to the Volkov wavefunction and we calculate ionization rates resulting from the correction in Sect. 3. There we also give an example showing that our method leads to results closer to other well-known theoretical calculations. There are concluding remarks in Sect. 4.

\section{KFR theory in finite spatial region}

Unlike in [1], only the laboratory frame of reference is utilized in this paper. We consistently use atomic units (a.u.) here: $\hbar=e=m_{\mathrm{e}}=1$, substituting explicitly -1 for the electronic charge. We keep any electric charge $Z$ of the nucleus in all the equations given below, but finally, in all the numerical calculations, we put $Z=1$, like for the hydrogen atom. Let us consider a wave function which is the exact solution of the following time-dependent Schrödinger equation (TDSE):

$$
\mathrm{i} \frac{\partial}{\partial t} \Psi(\boldsymbol{r}, t)=\left[\frac{1}{2}\left(-\mathrm{i} \boldsymbol{\nabla}+\frac{1}{c} \boldsymbol{A}(t)\right)^{2}-\frac{Z}{r}\right] \Psi(\boldsymbol{r}, t),
$$

where $\boldsymbol{A}(t)$ is the vector potential of the laser field (in the dipole approximation) and $r$ denotes the distance of the electron from the center of the atom (or ion). In the $S$-matrix theory of photoionization one uses stationary solutions of the TDSE with positive energy $E=\boldsymbol{p}^{2} / 2>0$ and $\boldsymbol{p}$ - the asymptotic (when $r \rightarrow \infty$ ) momentum as a parameter. Since one cannot solve Eq. (1) analytically, it is rather difficult to take something for granted in the general case, particularly if neither the laser field nor the Coulomb one can be treated perturbatively. However, for sufficiently strong laser field one can approximate the solution of Eq. (1) by the solution of the following equation:

$$
\mathrm{i} \frac{\partial}{\partial t} \Psi^{\mathrm{V}}(\boldsymbol{r}, t)=\frac{1}{2}\left(-\mathrm{i} \boldsymbol{\nabla}+\frac{1}{c} \boldsymbol{A}(t)\right)^{2} \Psi^{\mathrm{V}}(\boldsymbol{r}, t) .
$$

This solution is the well-known non-relativistic Volkov wave function (where $\boldsymbol{p}$ is a parameter)

$$
\Psi_{\boldsymbol{p}}^{\mathrm{V}}(\boldsymbol{r}, t)=\frac{1}{(2 \pi)^{3 / 2}} \exp \left(\mathrm{i} \boldsymbol{p} \boldsymbol{r}-\frac{\mathrm{i}}{2} \int_{-\infty}^{t}\left(\boldsymbol{p}+\frac{1}{c} \boldsymbol{A}(\tau)\right)^{2} \mathrm{~d} \tau\right) .
$$

Substituting Eq. (3) into Eq. (1) one obtains

$$
\frac{1}{2}\left(\boldsymbol{p}+\frac{1}{c} \boldsymbol{A}(t)\right)^{2} \Psi_{\boldsymbol{p}}^{\mathrm{V}}(\boldsymbol{r}, t) \approx\left[\frac{1}{2}\left(\boldsymbol{p}+\frac{1}{c} \boldsymbol{A}(t)\right)^{2}-\frac{Z}{r}\right] \Psi_{\boldsymbol{p}}^{\mathrm{V}}(\boldsymbol{r}, t) .
$$

One should expect, looking at Eq. (4), that the above approximate equality improves when either $\boldsymbol{p}$ or the amplitude of $\boldsymbol{A}(t)$ increases. The canonical momentum $\boldsymbol{p}$ in Eqs. (3) and (4) differs from the kinetic momentum $\boldsymbol{\pi}(t)=\boldsymbol{p}+\boldsymbol{A}(t) / c$, and they coincide only when $\boldsymbol{A}(t)=\mathbf{0}$. (In the velocity gauge KFR theory $[7,8]$ one 
assumes that $\lim _{t \rightarrow \pm \infty} \boldsymbol{A}(t)=\mathbf{0}$.) In photoelectron energy spectra, due to selection rules for angular momentum, one obtains the greatest multiphoton partial ionization rates (above-threshold ionization peaks) for some values of the final kinetic energy $E=p^{2} / 2$ of the outgoing electron. These values depend on the kind of polarization of the laser field and on its intensity. For linear polarization the low-energy electrons always dominate, but for circular polarization electrons with much higher energy $E=p^{2} / 2 \approx U_{\mathrm{P}}=I / 2 \omega^{2}$ usually dominate ( $U_{\mathrm{P}}$ is the ponderomotive potential and $I, \omega$ are the laser intensity and frequency) [11]. Therefore for the hydrogen atom, particularly for circular polarization and high intensity of the laser field, for most ionized electrons Eq. (4) is very well satisfied. In other words, these electrons appear well in the continuum of ionized states and the Volkov wave function is a very good approximation to the exact solution of Eq. (1). Much the same, the Coulomb-Volkov wave function derived by Reiss and Krainov [1]:

$$
\begin{aligned}
& \Psi_{\boldsymbol{p}}^{\mathrm{CV}}(\boldsymbol{r}, t)=\Psi_{\boldsymbol{p}}^{\mathrm{V}}(\boldsymbol{r}, t) \exp \left(\frac{\mathrm{i} Z}{\alpha_{0}} t\right) \\
& \quad=\frac{1}{(2 \pi)^{3 / 2}} \exp \left(\mathrm{i} \boldsymbol{p} \boldsymbol{r}-\frac{\mathrm{i}}{2} \int_{-\infty}^{t}\left(\boldsymbol{p}+\frac{1}{c} \boldsymbol{A}(\tau)\right)^{2} \mathrm{~d} \tau+\frac{\mathrm{i} Z}{\alpha_{0}} t\right)
\end{aligned}
$$

(where $\alpha_{0}$ is the radius of the circular classical motion of a free electron in the circularly polarized laser field) is also quite good approximation to the exact solution of Eq. (1). Substituting Eq. (5) into Eq. (1) one obtains

$$
\begin{aligned}
& {\left[\frac{1}{2}\left(\boldsymbol{p}+\frac{1}{c} \boldsymbol{A}(t)\right)^{2}-\frac{Z}{\alpha_{0}}\right] \Psi_{\boldsymbol{p}}^{\mathrm{CV}}(\boldsymbol{r}, t)} \\
& \quad \approx\left[\frac{1}{2}\left(\boldsymbol{p}+\frac{1}{c} \boldsymbol{A}(t)\right)^{2}-\frac{Z}{r}\right] \Psi_{\boldsymbol{p}}^{\mathrm{CV}}(\boldsymbol{r}, t) .
\end{aligned}
$$

The improvement due to the appearance of the term $-Z / \alpha_{0}$ on the left-hand side in the approximate equality is substantial only for very large $r$, because Reiss and Krainov have assumed that $\alpha_{0} \gg 1 / Z$ [1]. The Coulomb correction $-Z / \alpha_{0}$ may be very small due to large $\alpha_{0}$ and the correction tends to zero for very intense fields, because $\alpha_{0} \propto \sqrt{I}$.

Let us consider the influence of the distance of an ionized electron from the center of an atom (or ion) on ionization rate. Within the limits of the $S$-matrix theory of photoionization we can do it in the following way. The general analytical expressions for ionization rate of the spherically symmetric initial state in the strong-field approximation (SFA) $[6,7]$ are

$$
\begin{aligned}
& \Gamma_{\text {cir }}^{\mathrm{SFA}}=(2 \pi)^{2} \sum_{n=n_{0}}^{\infty} p_{n}\left[\tilde{\Phi}_{i}\left(p_{n}\right)\left(\frac{p_{n}^{2}}{2}+E_{\mathrm{B}}\right)\right]^{2} \\
& \times \int_{0}^{\pi} \mathrm{d} \vartheta \sin \vartheta J_{n}^{2}\left(\sqrt{\frac{2 z}{\omega}} p_{n} \sin \vartheta\right)
\end{aligned}
$$




$$
\begin{aligned}
& \Gamma_{\mathrm{lin}}^{\mathrm{SSA}}=(2 \pi)^{2} \sum_{n=n_{0}}^{\infty} p_{n}\left[\tilde{\Phi}_{i}\left(p_{n}\right)\left(\frac{p_{n}^{2}}{2}+E_{\mathrm{B}}\right)\right]^{2} \\
& \times \int_{0}^{\pi} \mathrm{d} \vartheta \sin \vartheta J_{n}^{2}\left(2 \sqrt{\frac{z}{\omega}} p_{n} \cos \vartheta,-\frac{z}{2}\right),
\end{aligned}
$$

for circular and linear polarization respectively, where $p_{n}^{2} / 2=(n-z) \omega-E_{\mathrm{B}}$ is the final kinetic energy of the outgoing electron and its binding energy in the initial state is $E_{\mathrm{B}}$. (In Eqs. (7) one does not necessary assume that $E_{\mathrm{B}}=Z^{2} / 2$.) If the state is the $\mathrm{H}(1 s)$ atom $\tilde{\Phi}_{i}(p)$ (in the momentum space, normalized to unity in the entire space), then $E_{\mathrm{B}}=Z^{2} / 2=0.5$ a.u. (We refer the reader to our recent paper [12] and to [7] for more details regarding Eqs. (7).) The expressions (7) have been obtained after integration over all possible positions $\boldsymbol{r}$ of the electron in space (when calculating the $S$-matrix element). This means that all possible electron distances from the center of an atom (or ion) $0 \leq r \leq \infty$ contribute to ionization rates in Eqs. (7). Let us check what is indeed the maximum distance $R$ of the electron from the center of an atom (or ion), which really contributes to ionization rates in Eqs. (7). To this end we have derived expressions analogical to Eqs. (7), but with $0 \leq r \leq R$. To achieve this instead of the initial state wave function $\Phi_{i}(r)$ one can use the following "wave function":

$$
\Phi_{R}(r)=\Phi_{i}(r)[1-\theta(r-R)]=\sqrt{\frac{Z^{3}}{\pi}} \exp (-Z r)[1-\theta(r-R)]
$$

$(\theta(x)$ is the Heaviside step function), which does not have to be normalized to unity in the entire space or obey exactly everywhere in space the time-independent Schrödinger equation. In fact, Eq. (8a) only shows that in the calculation of ionization rate the distances $0 \leq r \leq R$ from the ionic core have been taken into account. Equation (8a) leads to

$$
\begin{gathered}
\tilde{\Phi}_{R}(p)=\int \frac{\mathrm{d}^{3} r}{(2 \pi)^{3 / 2}} \Phi_{R}(r) \exp (-\mathrm{i} \boldsymbol{p} \boldsymbol{r})=\frac{\sqrt{2 Z^{3}}}{\pi p\left(p^{2}+Z^{2}\right)^{2}}\left\{2 Z p+\mathrm{e}^{-Z R}\right. \\
\left.\times\left[\left(p^{2}-p^{2} Z R-Z^{2}-Z^{3} R\right) \sin p R-\left(p^{3} R+2 p Z+p Z^{2} R\right) \cos p R\right]\right\} .
\end{gathered}
$$

(From Eq. (8b) one obtains the well-known expression for $\tilde{\Phi}_{i}(p)$ in the limit $R \rightarrow \infty$.) Replacing $E_{\mathrm{B}}=Z^{2} / 2$ by $E_{\mathrm{B}}^{\prime}=Z^{2} / 2-Z / \alpha_{0}$ in Eq. (7a) one obtains the so-called Coulomb corrected strong-field approximation (CSFA) [1]. In Fig. 1 we plot the CSFA ionization rate of the $\mathrm{H}(1 s)$ atom as a function of intensity of the laser field for $\omega=0.074$ a.u. $(\lambda=616 \mathrm{~nm}$ - a typical optical wavelength $)$ and a few different $R$ 's (thus $\tilde{\Phi}_{i}(p)$ is replaced by Eq. (8b) in Eq. (7a)). The range of intensities corresponds to 10 a.u. $\leq \alpha_{0} \leq 100$ a.u. in Fig. 1. Let us have a look at consecutive curves on this graph. It is obvious that certainly neither $R=2$ a.u. nor $R=4$ a.u. can properly describe ionization rates for all intensities shown here. We have also checked that even for a few intensities, where ionization rates are equal with those of $R=\infty$ (see the intersection points of the $R=2$ a.u. and $R=4$ a.u. curves with the $R=\infty$ one in Fig. 1) the photoelectron 


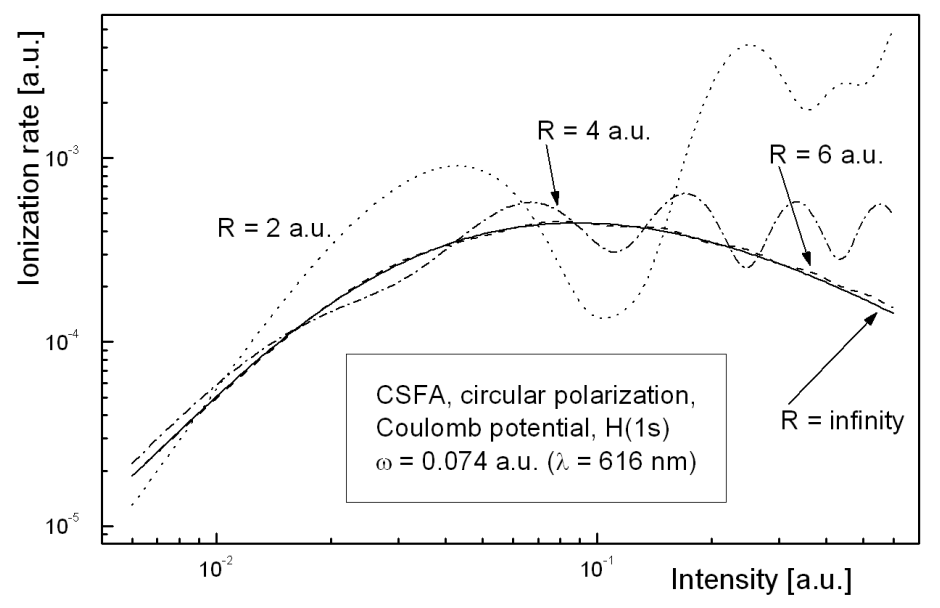

Fig. 1. The CSFA ionization rate (of the $\mathrm{H}(1 s)$ atom) as a function of intensity for $\omega=0.074$ a.u. in the range of intensities corresponding to 10 a.u. $\leq \alpha_{0} \leq 100$ a.u. for the circularly polarized laser field. The solid line $(R=\infty)$ is the Reiss-Krainov result [1]. The other three ionization rates have been calculated assuming that only $0 \leq r \leq R$ contribute to the $S$-matrix element (respectively, for $R=2,4,6$ a.u.).

energy spectra are significantly deformed. One needs at least about $R=6$ a.u. to properly reproduce the $R=\infty$ result for the laser field parameters shown in Fig. 1. Moreover, the limiting value of $R$, which reasonably describes ionization rate, grows with increasing intensity. What is even more interesting, in spite of the fact that the "wave function" (8a) is normalized to less than unity (for example, when $R=2$ a.u. one obtains $\left.\int \mathrm{d}^{3} r\left|\Phi_{R}(r)\right|^{2} \approx 0.762\right)$, for some intensities and for finite $R$ one obtains much greater ionization rate than the true $(R=\infty)$ CSFA result. It appears that the quantum-mechanical interference effect plays a very important role in the strong-field photoionization. For the highest intensities shown in Fig. 1 it is the destructive interference of different possible space positions $\boldsymbol{r}$ of ionized electron, roughly from $0 \leq r \leq 6$ a.u., that produces the true $(R=\infty)$ CSFA result.

What is the effect of finite $R$ for much higher intensities of the laser field? We show it for the SFA $\left(E_{\mathrm{B}}=Z^{2} / 2\right.$ in Eqs. (7)) in Figs. 2 and 3 for both circular and linear polarization, respectively. The range of intensities corresponds to limitations of the non-relativistic SFA in Figs. 2 and $3\left(1 \leq z_{1}\right.$ and $z_{f} \leq 0.1$, where $z_{1}=2 U_{\mathrm{P}} / E_{\mathrm{B}}$ and $z_{f}=2 U_{\mathrm{P}} / c^{2}$; see $[7,12]$ for more details). It appears that for circular polarization and laser fields strong enough $R=6$ a.u. or even $R=8$ a.u. are not sufficient to properly describe ionization rate in the SFA. In contrast, for linear polarization the assumption that only $r \lesssim 1$ a.u. contribute to ionization rate is quite well satisfied for the laser field parameters from Fig. 3.

In principle, the SFA has been introduced for short-range potentials [7] and it should work better in this case than for the long-range Coulomb potential. 


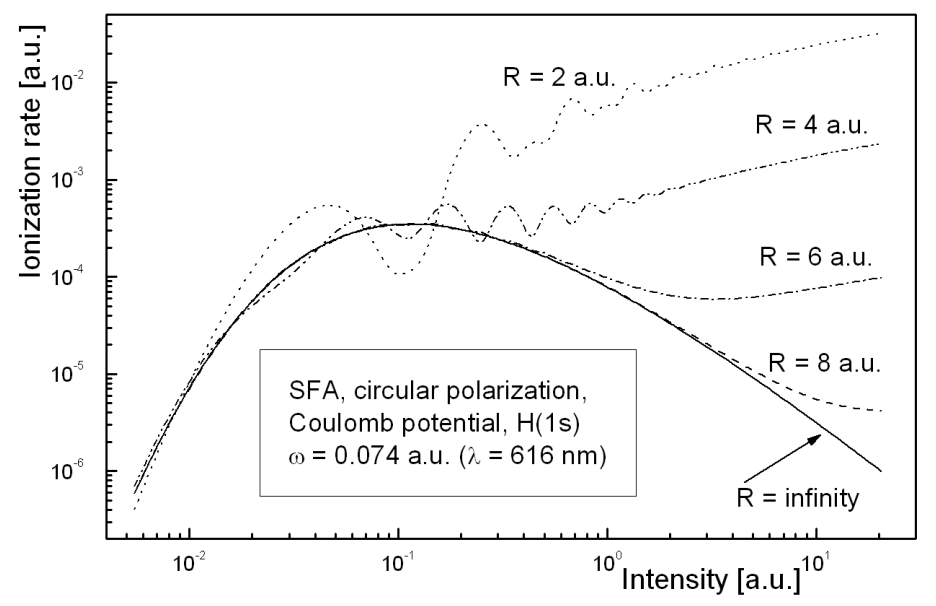

Fig. 2. The SFA ionization rate (of the $\mathrm{H}(1 s)$ atom) as a function of intensity for $\omega=0.074$ a.u. in the range of intensities corresponding to $1 \leq z_{1}$ and $z_{f} \leq 0.1$ for the circularly polarized laser field. The solid line $(R=\infty)$ is the Reiss result [7]. The other four ionization rates have been calculated assuming that only $0 \leq r \leq R$ contribute to the $S$-matrix element (respectively, for $R=2,4,6,8$ a.u.).

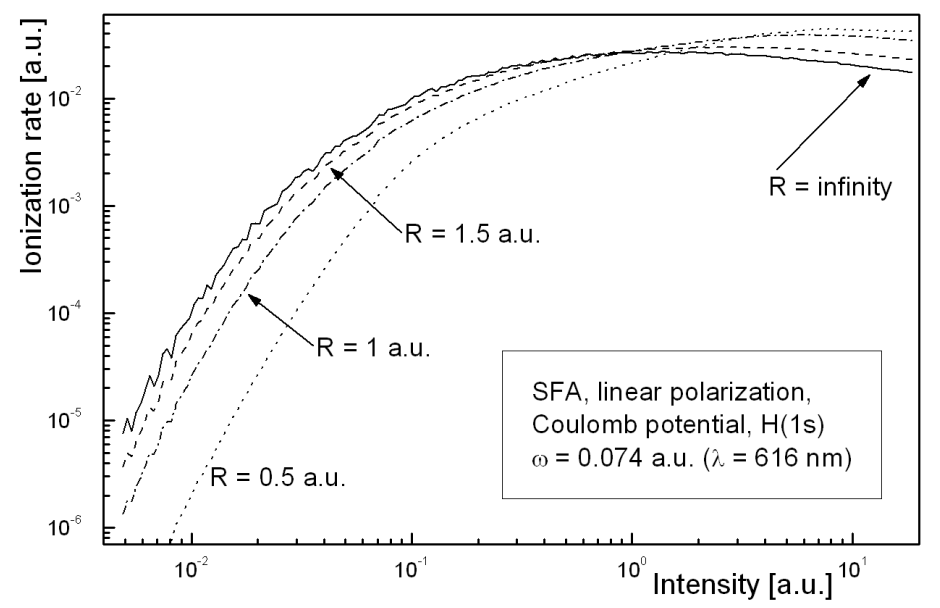

Fig. 3. The SFA ionization rate (of the $\mathrm{H}(1 s)$ atom) as a function of intensity for $\omega=0.074$ a.u. in the range of intensities corresponding to $1 \leq z_{1}$ and $z_{f} \leq 0.1$ for the linearly polarized laser field. The solid line $(R=\infty)$ is the Reiss result [7]. The other three ionization rates have been calculated assuming that only $0 \leq r \leq R$ contribute to the $S$-matrix element (respectively, for $R=0.5,1,1.5$ a.u.).

Therefore let us also check the effect of finite $R$ for the zero-range potential for the same binding energy $\left(E_{\mathrm{B}}=Z^{2} / 2\right)$ and laser field parameters as in the Coulomb potential case. On the analogy of Eqs. (8) we obtain 


$$
\begin{aligned}
& \Phi_{0 R}(r)=\Phi_{0}(r)[1-\theta(r-R)]=\sqrt{\frac{Z}{2 \pi}} \frac{1}{r} \exp (-Z r)[1-\theta(r-R)], \\
& \tilde{\Phi}_{0 R}(p)=\frac{\sqrt{Z}}{\pi\left(p^{2}+Z^{2}\right)}\left[1-\mathrm{e}^{-Z R}\left(\frac{Z}{p} \sin p R+\cos p R\right)\right],
\end{aligned}
$$

and we substitute Eq. (9b) into Eqs. (7). Also in this case, in the limit $R \rightarrow \infty$, one has the initial state wave function which is exact. Moreover, the SFA ionization rate for the zero-range potential is gauge-invariant, i.e. the length and velocity gauge results are identical $[12,13]$.

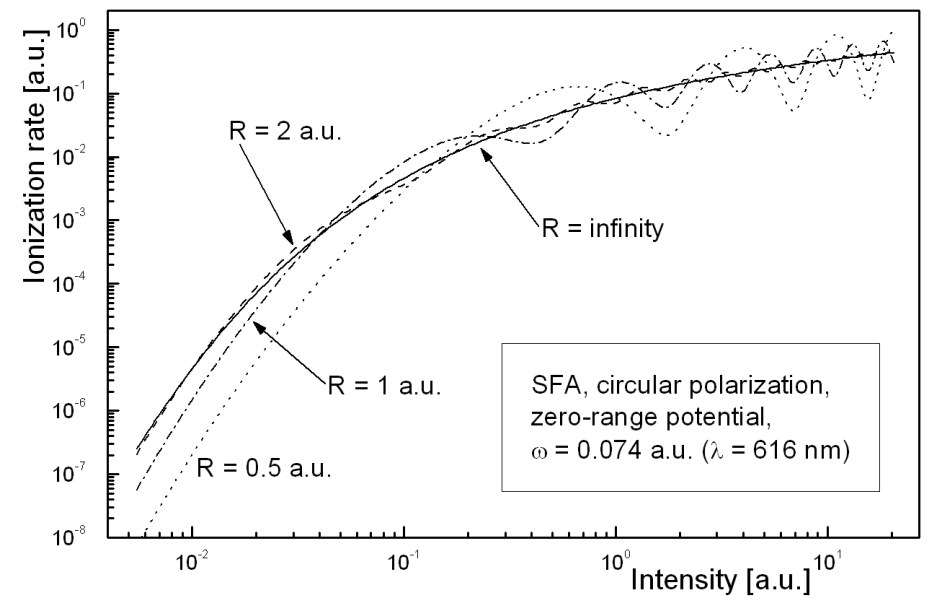

Fig. 4. The SFA ionization rate (of the only bound state in the zero-range potential with $E_{\mathrm{B}}=0.5$ a.u.) as a function of intensity for $\omega=0.074$ a.u. in the range of intensities corresponding to $1 \leq z_{1}$ and $z_{f} \leq 0.1$ for the circularly polarized laser field. The solid line $(R=\infty)$ is the result of Eq. (7a) (with the substitution of Eq. (9b) for $R \rightarrow \infty)$. The other three ionization rates have been calculated assuming that only $0 \leq r \leq R$ contribute to the $S$-matrix element (respectively, for $R=0.5,1,2$ a.u.).

In Figs. 4 and 5 we present ionization rate (for an electron bound by this potential) as a function of intensity for the circular and linear polarization of the laser field, respectively. It appears that for the zero-range potential and both polarizations ionization always takes place roughly inside the sphere of the radius $R \approx 2$ a.u. It follows from Fig. 5 that for linear polarization the radius of the sphere, where ionization takes place, decreases with increasing intensity. This is in good qualitative agreement with the evaluation given by Gribakin and Kuchiev in [13]. For example, for the following intensities: $I=0.01$ a.u., 0.1 a.u., and 1.0 a.u. one obtains $R=2.8$ a.u. 1.8 a.u. and 1.0 a.u., respectively, from Eq. (2) of [13]. (Let us note that in their paper the $\mathrm{H}^{-}$ion was considered with much smaller binding energy and therefore much larger $R$, but Eq. (1) of [13] is satisfied in our case, because $\omega=0.074$ a.u. $\ll 0.5$ a.u. $=E_{\mathrm{B}}$.) It follows from Figs. $2-5$ that the Coulomb potential case in the circularly polarized strong laser field is exceptional 


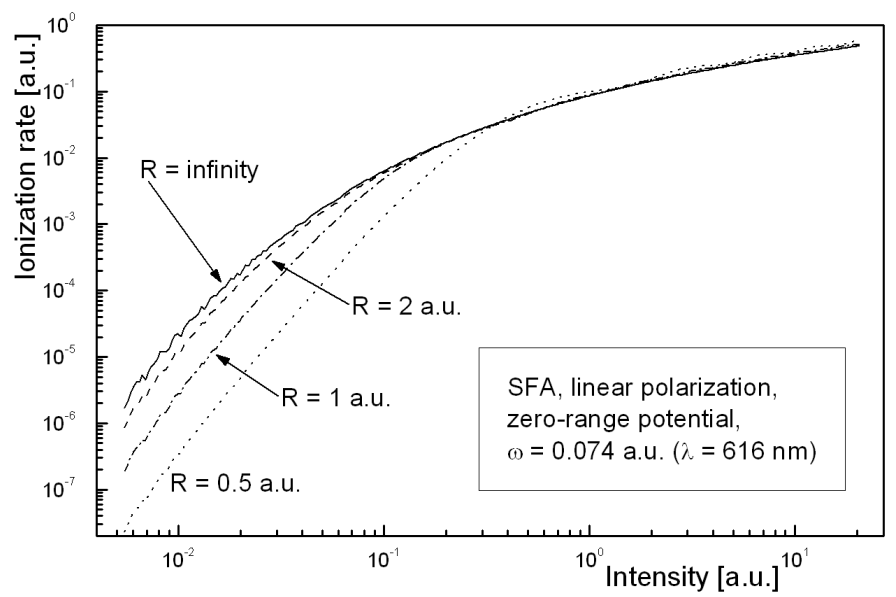

Fig. 5. The SFA ionization rate (of the only bound state in the zero-range potential with $E_{\mathrm{B}}=0.5$ a.u.) as a function of intensity for $\omega=0.074$ a.u. in the range of intensities corresponding to $1 \leq z_{1}$ and $z_{f} \leq 0.1$ for the linearly polarized laser field. The solid line $(R=\infty)$ is the result of Eq. (7b) (with the substitution of Eq. (9b) for $R \rightarrow \infty)$. The other three ionization rates have been calculated assuming that only $0 \leq r \leq R$ contribute to the $S$-matrix element (respectively, for $R=0.5,1,2$ a.u.).

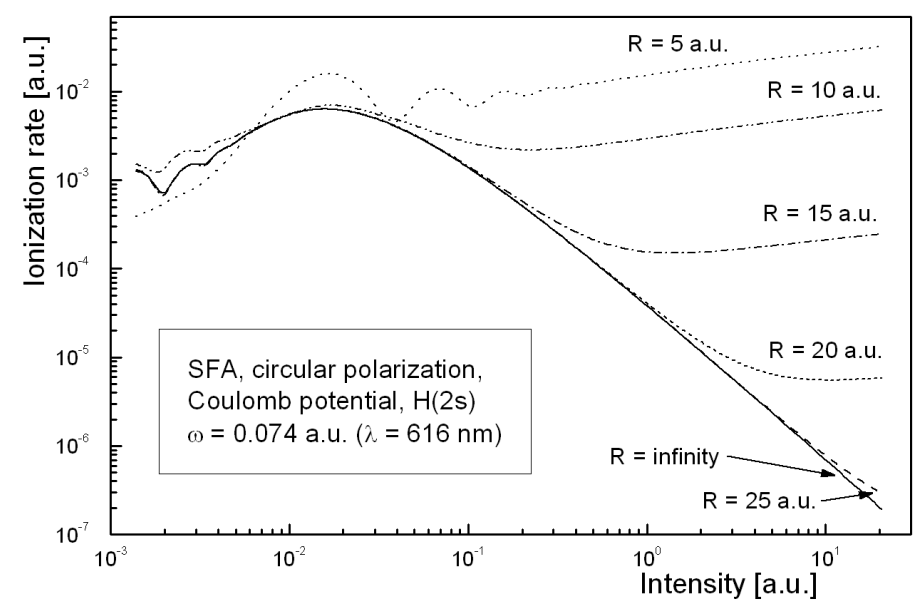

Fig. 6. The SFA ionization rate (of the $\mathrm{H}(2 s)$ atom) as a function of intensity for $\omega=0.074$ a.u. in the range of intensities corresponding to $1 \leq z_{1}$ and $z_{f} \leq 0.1$ for the circularly polarized laser field. The solid line $(R=\infty)$ is the Reiss result [7]. The other five ionization rates have been calculated assuming that only $0 \leq r \leq R$ contribute to the $S$-matrix element (respectively, for $R=5,10,15,20,25$ a.u.).

in a way because photoionization takes place in much larger space than in the remaining three cases. 
In Fig. 6 we show the SFA ionization rate of the $\mathrm{H}(2 s)$ atom as a function of intensity for $\omega=0.074 \mathrm{a}$.u. in the range of intensities corresponding to $1 \leq z_{1}$ and $z_{f} \leq 0.1$ for the circularly polarized laser field (since for the first excited state of the hydrogen atom $E_{\mathrm{B}}=Z^{2} / 8=0.125$ a.u., the condition $z_{1}=1$ corresponds to lower intensity than in Figs. 1-5). For this initial state, which we treat analogically, below we only give its momentum space "wave function" for finite $R$ (Eq. (10) is analogical to Eqs. (8b) and (9b), and here one obtains the exact $\tilde{\Phi}_{i}(p)$ for the $\mathrm{H}(2 s)$ atom in the limit $R \rightarrow \infty)$ :

$$
\begin{aligned}
\tilde{\Phi}_{R}( & p)=\frac{Z^{3 / 2}}{4 \pi p} \int_{0}^{R} \mathrm{~d} r\left(2 r-Z r^{2}\right) \mathrm{e}^{-Z r / 2} \sin p r=\frac{Z^{3 / 2}}{2 \pi p\left(4 p^{2}+Z^{2}\right)^{3}} \\
& \times\left\{128 p^{3} Z-32 p Z^{3}+\exp (-Z R / 2)\left[\operatorname { s i n } p R \left(64 p^{4}-96 p^{4} R Z\right.\right.\right. \\
& \left.-96 p^{2} Z^{2}+16 p^{4} R^{2} Z^{2}-16 p^{2} R Z^{3}+4 Z^{4}+8 p^{2} R^{2} Z^{4}+2 R Z^{5}+R^{2} Z^{6}\right) \\
& +\cos p R\left(-64 p^{5} R-128 p^{3} Z+32 p^{5} R^{2} Z+32 p^{3} R Z^{2}\right. \\
& \left.\left.\left.+32 p Z^{3}+16 p^{3} R^{2} Z^{3}+12 p R Z^{4}+2 p R^{2} Z^{5}\right)\right]\right\}
\end{aligned}
$$

It follows from Fig. 6 that the spatial region that really contributes to ionization rate expands from about $R=10 \div 15$ a.u. to $R \approx 25$ a.u. with increasing intensity. Of course, this is connected with the fact that the $\mathrm{H}(2 s)$ atom is larger than the $\mathrm{H}(1 s)$ atom (for instance, $\langle r\rangle=3 / 2$ a.u. for $\mathrm{H}(1 s)$, and $\langle r\rangle=6$ a.u. for $\mathrm{H}(2 s)$ ).

\section{Coulomb correction to the Volkov wave function}

Let us now treat these ionization rates for finite $R$ as a hint for finding a better Coulomb correction in the SFA ionization rate formula for circular polarization (Eq. (7a) with $R=\infty$ ). Figures 1, 2 and 6 suggest that instead of the $-Z / \alpha_{0}$ Coulomb correction in Eq. (6) one could use $-Z / R_{\text {eff }}(\omega, I)$ to compensate partially the term $-Z / r$ on the right-hand side of this equation. The effective (or phenomenological) parameter $R_{\text {eff }}$ would be a certain function of the laser frequency and intensity (however, we have numerically checked that the dependence of $R_{\text {eff }}$ on frequency is very weak for 0.01 a.u. $<\omega<1$ a.u. and one can use the same $R_{\text {eff }}$ as for $\omega=0.074$ a.u.). Therefore the Volkov wave function would be multiplied not by $\exp \left(\mathrm{i} Z t / \alpha_{0}\right)$, but rather by $\exp \left(\mathrm{i} Z t / R_{\text {eff }}\right)$ in Eq. (5). Let us stress again (which is particularly visible in Figs. 1, 2 and 6 ) that there is no electron-nucleus distance, around which (in a small volume) the strong-field ionization in the circularly polarized laser field is well localized. Always at least a few atomic units is necessary "to produce" the SFA ionization rate. The region $r \lesssim 1 / Z$ is indeed very important for the transition matrix element, but this is not the only important region. If we substitute the Volkov wave function multiplied by $\exp \left(\mathrm{i} Z t / R_{\text {eff }}\right)$ into Eq. (1) (for the fixed $\omega, I$ and $\boldsymbol{p}$ ) the relative error on the left-hand of this equation will depend on both $\boldsymbol{r}$ and $t$, and the error will be proportional to $\left|Z / r-Z / R_{\text {eff }}\right|$. Therefore for very small electron-nucleus distances $r$ our Coulomb correction improves accuracy of the Volkov wave function 
only slightly. However, larger electron-nucleus distances in number dominate and for $r \gtrsim 1 / Z$ the above mentioned relative error decreases considerably. For that reason we expect that utility of our Coulomb correction should grow with the size of an atom (for example with the principal quantum number $n$ for excited states of $\mathrm{H}(n s))$.

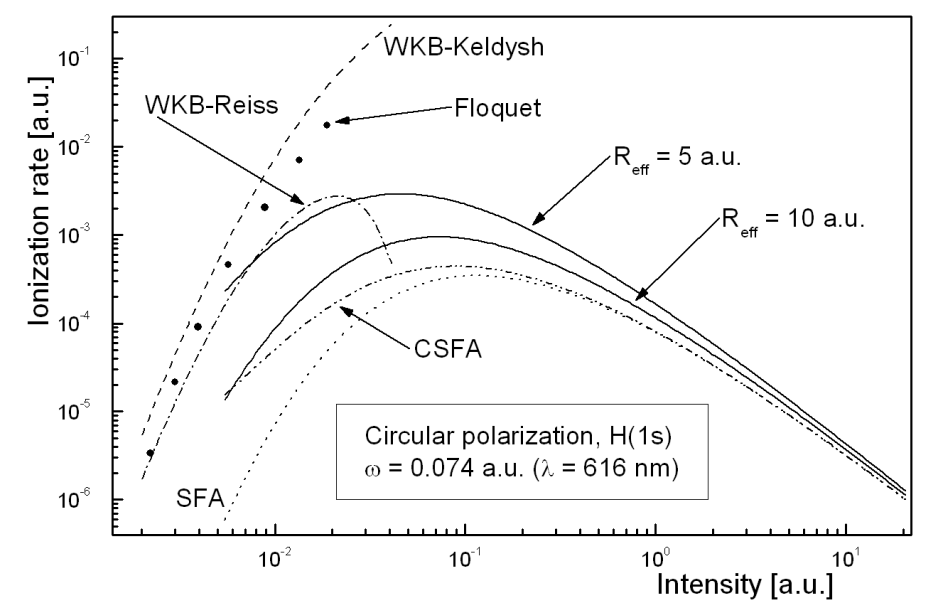

Fig. 7. Various theoretical ionization rates (of the $\mathrm{H}(1 s)$ atom) as a function of intensity for $\omega=0.074$ a.u. and the circularly polarized laser field (see text for details).

As a result, in the SFA ionization rate formula the binding energy (for the $\mathrm{H}(1 s)$ atom) would be replaced by $E_{\mathrm{B}}^{\prime}=Z^{2} / 2-Z / R_{\text {eff. }}$. In Fig. 7 we show such ionization rates (by two identical solid lines) as a function of intensity for the circularly polarized laser field for two different constant values $R_{\text {eff }}=5$ a.u. and $R_{\text {eff }}=10$ a.u. Roughly these values are suggested by Fig. 2 for $\omega=0.074$ a.u. as the limits between which our new Coulomb-corrected SFA ionization rate could run across. (For the lowest intensities shown in Fig. $2 R_{\text {eff }} \approx 5$ a.u., and for the highest intensities $-R_{\text {eff }} \approx 10$ a.u.) For comparison we also show the SFA and the CSFA ionization rates in Fig. 7. In the figure there are also some other theoretical calculations (analytical formulae were derived by other authors), which are valid for smaller intensities, but which have some common range of validity with the above mentioned various strong-field calculations. The Floquet calculations have been taken from Fig. 5 of [14]. The WKB Coulomb corrected KFR theory $[15,16,12,17]$, in both gauges, has a high-intensity limit connected with existence of the Coulomb barrier and the critical laser field strength [14] in the $\mathrm{H}(1 s)$ atom. The WKB-Reiss ionization rate has been calculated from Eq. (9a) of [17] and the WKB-Keldysh one from Eq. (32a) of [12]. One can easily observe that in Fig. 7, around $I=0.01$ a.u., the curve with $R_{\text {eff }}=5$ a.u. is much closer to the Floquet and the WKB Coulomb corrected KFR results than the SFA and CSFA curves. 


\section{Concluding remarks}

In conclusion, the main result of the present paper is revealing that photoionization takes place in much greater volume than one could expect $(r \lesssim 1 / Z)$, if the following three conditions are simultaneously satisfied: (i) the binding potential is the (long-range) Coulomb one, (ii) the laser field is strong enough, and (iii) the laser field is circularly polarized. For the zero-range potential (for both polarizations) or for the Coulomb potential (when the laser field is linearly polarized) the ionization takes place inside the sphere of the radius of about 2 a.u. (if $Z=1$ and $E_{\mathrm{B}}=Z^{2} / 2$ ). This is another explanation why the SFA should work much better for the circular polarization than for the linear one, if the ionized electron interacts with the Coulomb potential. In contrast, when the outer electron interacts with the short-range (or zero-range, in theory) potential, like in $\mathrm{H}^{-}$ion, the SFA is also a very good approximation, because the binding energy is much lower and the initial-state wave function is much more extended in space than in the atomic case.

One should stress that our results for the Coulomb potential (unlike for the zero-range potential) may be gauge-dependent. Analogous calculations (with finite $R$ instead of $R=\infty$ ) in the length gauge would be much more difficult. However, it follows from the general form of the $S$-matrix element in the KFR theory that great electron-nucleus distances can be even more significant (for the calculated ionization rate) in the length gauge than in the velocity gauge (see also [13]). For a given frequency and an intensity of the laser field, with the help of our method, one can always approximately find the parameter $R_{\text {eff }}(\omega, I)$. The parameter would be the smallest finite $R$ such that the ionization rate and the photoelectron energy spectrum is nearly identical with the ordinary KFR result (with $R=\infty$ ). Our Coulomb correction is not univocal and cannot be expressed by a simple analytical formula (like the Reiss-Krainov result [1]). For example, the simplest choice for the hydrogen atom is the following binding energy: $E_{\mathrm{B}}^{\prime}=E_{\mathrm{B}}-1 / R_{\text {eff }}$, where $R_{\text {eff }}=10$ a.u. for $\mathrm{H}(1 s)$ and $R_{\text {eff }}=25$ a.u. for $\mathrm{H}(2 s)$. Our Coulomb correction always results in a greater and more accurate ionization rate.

\section{Acknowledgments}

The author is indebted to Professor Piotr Kosiński for useful remarks and interesting discussions concerning this paper and to Professor Howard R. Reiss for his correspondence on the present subject. This paper was supported by the University of Łódź.

\section{References}

[1] H.R. Reiss, V.P. Krainov, Phys. Rev. A 50, R910 (1994).

[2] W. Gordon, Z. Phys. 40, 117 (1926).

[3] D.M. Volkov, Z. Phys. 94, 250 (1935). 
[4] L.V. Keldysh, Zh. Eksp. Teor. Fiz. 47, 1945 (1964) [Sov. Phys. JETP 20, 1307 (1965)].

[5] A.M. Perelomov, V.S. Popov, M.V. Terent'ev, Zh. Eksp. Teor. Fiz. 50, 1393 (1966) [Sov. Phys. JETP 23, 924 (1966)].

[6] F.H.M. Faisal, J. Phys. B 6, L89 (1973).

[7] H.R. Reiss, Phys. Rev. A 22, 1786 (1980).

[8] H.R. Reiss, Prog. Quant. Electron. 16, 1 (1992).

[9] H.R. Reiss, Laser Phys. 7, 543 (1997).

[10] H.R. Reiss, Phys. Rev. D 11, 388 (1975).

[11] H.R. Reiss, Phys. Rev. A 54, R1765 (1996).

[12] J. Bauer, Phys. Rev. A 73, 023421 (2006).

[13] G.F. Gribakin, M.Yu. Kuchiev, Phys. Rev. A 55, 3760 (1997).

[14] R. Shakeshaft, R.M. Potvliege, M. Dörr, W.E. Cooke, Phys. Rev. A 42, 1656 (1990).

[15] V.P. Krainov, J. Opt. Soc. Am. B 14, 425 (1997).

[16] V.P. Krainov, B. Shokri, Zh. Eksp. Teor. Fiz. 107, 1180 (1995) [Sov. Phys. JETP 80, 657 (1995)].

[17] J.H. Bauer, Phys. Rev. A 75, 045401 (2007). 